\title{
LA NUEVA GENERACIÓN DE PLÁSTICOS: UN CUADRO DE INNOVACIÓN
}

\author{
Andrea Larson, Karen Peabody O’Brien y Alia Anderson
}

\section{RESUMEN:}

La historia de los plásticos ha sido impulsada por una serie de fuerzas que han motivado permanentemente la innovación. Entre ellas se encuentran consideraciones económicas, preocupaciones ambientales, problemas de salud pública y presiones políticas.

El artículo repasa el sentido de estas fuerzas y muestra cómo los innovadores han sabido responder a las diversas presiones y han agudizado su inventiva para desarrollar otras posibilidades. En un interesante y completo recorrido se hace un repaso de la historia, en el que se mencionan logros como el reemplazo marfil de las bolas de billar por plástico, la vinculación de estos materiales a las carrocerías de automóviles y el reemplazo de sustancias que tradicionalmente hacen parte de ellos para responder de manera más eficiente a las presiones ambientales.

Pese a la historia creativa de este maravilloso material, los empresarios de hoy enfrentan muchos más desafíos. Uno de ellos se deriva de las preocupaciones en torno a los plásticos derivados de combustibles fósiles, en un momento en que el precio del petróleo sigue aumentando. Otro, la necesidad de obtener de nuevos materiales que no afecten la salud de los usuarios y sean más amigables con el medio ambiente. Pero estas presiones, como se indica en el artículo, no son más que estímulos para encontrar nuevas alternativas. "Las innovaciones que vemos ahora en la industria de los plásticos representan la historia clásica del empresario, en la que las compañías líderes e individuos ven oportunidades donde otros sólo ven problemas".

\section{PALABRAS CLAVE}

Plásticos, resina plastica sintética, Bakelita, toxinas, bistenol A, aneuplodia, ftalatos, ácido polisáctico o PLA, Poliolefinas, PVC.

\section{ABSTRACT:}

The history of plastics has been boosted by a series of forces that have constantly encouraged innovation. Among them are economic considerations, environmental concerns, public health issues, and political pressure.

The article overviews the sense of these forces and shows how innovators have been able to put up with the various pressures, and have sharpened their creativity to give rise to other alternatives. In an interesting and comprehensive overview, a review of history is made, in which some achievements are mentioned, like the replacement of marble by plastic in pool balls, the use of this material in car frame manufacturing, and the replacement of substances traditionally used in this manufacturing process, to efficiently meet environmental concerns.

In spite of the history of innovation of this wonderful material, today's entrepreneurs face many challenges. One of them results from concerns with respect to plastics derived from fossil oils, at a time when oil prices keep rising. Another one is the need for finding new materials which do not affect consumers' health and which are more environmentally friendly. But these pressures, as pointed out in the article, are nothing less than incentives to find new alternatives. "Innovations being introduced in the plastics industry represent the traditional history of the entrepreneur in which leading companies and individuals see opportunities where others just see problems." 
INVIERNO 2005 • CONFERENCIAS BATTEN

Una discusión acerca de la innovación, el espiritu empresarial y la transformación corporativa.

La historia de la invención y desarrollo del plástico es una historia acerca de algunas de las fuerzas más poderosas en innovación: consideraciones económicas, preocupaciones ambientales y de salud pública, y presiones políticas. La historia de los plásticos, por supuesto, no ha concluido. Las fuerzas cambian, los innovadores responden con productos y tecnologías revolucionarias, y estos, a su turno, dan pie a circunstancias y presiones completamente nuevas, nuevas oportunidades para la invención.

¿En qué parte de la historia estamos? Las innovaciones actuales en los plásticos, y en muchas otras industrias, están siendo impulsadas por preocupaciones frente a la sostenibilidad; por crear productos que cumplan con metas económicas, ambientales, y sociales. Estas preocupaciones, que impulsan

\section{RESEÑA AUTOR:}

Andrea Larson es profesora asociada en la Escuela Darden ${ }^{1}$, Karen Peabody O'Brien es investigadora asociada en el Green Chemistry Institute. ${ }^{2}$ Alia Anderson es asistente de investigación en la Escuela Darden.

Parte de la información del presente artículo provino de las memorias de un taller sobre Innovación en Plásticos en septiembre de 2004 llevado a cabo en la Escuela Darden. Para mayor información acerca de este evento, por favor póngase en contacto con los autores. el desarrollo de celdas combustibles, autos híbridos, productos de alimentos orgánicos, y energía eólica, para nombrar unos pocos, también inspiran a los pioneros a encontrar alternativas químicamente benignas de alto desempeño, a producir plásticos basados en combustibles fósiles. Antes de describir cómo algunos innovadores en plásticos están respondiendo al imperativo de la sostenibilidad, consideremos brevemente las fuerzas que dieron vida a los plásticos y aquellas que están funcionando hoy.

\section{LAS FUERZAS PROMOTORAS DE LA INNOVACIÓN}

La resina plástica sintética fue inventada a finales del siglo diecinueve, cuando la presión social y financiera contra el comercio de marfil motivó a los innovadores a diseñar un material sustituto. Según el libro American Plastic: A Cultural History de Jeffrey Meikle ${ }^{3}$, en 1863 una firma manufacturera ofreció $\$ 10,000$ a cualquiera que pudiera inventar un reemplazo aceptable para el marfil en las bolas de billar. Después de una larga investigación, un impresor americano descubrió que una combinación de pulpa de algodón (celulosa), nitratos, y alcanfor podía moldearse en una sustancia durable, brillante, distinta de cualquier otro material.

El siguiente salto hacia adelante en el diseño de plásticos se debió a un descubrimiento accidental de Leo Baekeland, quien

1 Puede ser localizada en LarsonA@darden.virginia.edu.

2 Comuníquese con ella en ObrienK@darden.virginia.edu.

3 Jeffery Meikle, author of American Plastic: A Cultural History, discusses the paradoxical relationship between Americans and plastics. On one hand, Americans are driven by a desire for authenticity which causes plastics to be repulsive as inauthentic. On the other hand, plastics have enchanted Americans. Their malleability seems to be the stuff of a new utopia. American Plastic: A Cultural History, Rutgers University Press, 1996. Jeffery Meikle, autor de American Plastic: A Cultural History discute acerca de la paradójica relación entre los plásticos y los norteamericanos. Por una parte, los norteamericanos se dejan llevar por el deseo de ser auténticos generando una repulsión hacia los plásticos que no lo son. Por otra parte, los plásticos han encantado a los norteamericanos. La maleabilidad es la sustancia de una nueva utopia. American Plastic: A Cultural History, Rutgers University Press, 1996. 
en 1910 estaba buscando una alternativa al barniz de laca. Derivado de una secreción que dejan los cucarrones en ciertos árboles en el sur de Asia, el barniz de laca es un aislante eléctrico efectivo. A comienzos del siglo veinte, el papel impregnado de este barniz era usado para aislar cables eléctricos, y a medida que la electrificación se extendió, la demanda de barniz de laca pronto superó a la oferta. Al intentar crear una alternativa, Baekeland descubrió que combinando fenol y formaldehído bajo condiciones particulares de calor y presión, producía una sustancia totalmente nueva, moldeable y durable. Él describió su producto revolucionario de manera precisa -plástico baquelita- "el material de los mil usos."

Otro de los primeros innovadores en plásticos fue Henry Ford, quien en 1941 presentó una carrocería de auto hecha de un plástico que era una combinación de trigo, paja, y soya. Este auto "de cultivo" tenía sentido después de la Depresión y de una sequía que había llevado a los agricultores americanos al endeudamiento. Los plásticos basados en plantas también tenían sentido a comienzos de la Segunda Guerra Mundial, que acrecentó la incertidumbre acerca del acceso a recursos del extranjero. Sin embargo, el final de la guerra trajo sin impedimentos el petróleo extranjero y el posterior nacimiento de la edad de los petroquímicos. En las décadas posteriores, las constantes innovaciones en la tecnología química y en las técnicas de producción han hecho que se quede muy corta la declaración de Baekeland: nosotros dependemos ahora de los plásticos para la elaboración de materia prima, la producción, los empaques, y el transporte de casi todos los productos que usamos.
Las preocupaciones acerca de la disponibilidad de recursos naturales todavía impulsa la innovación en los plásticos. Mientras la invención del plástico era una respuesta a la escasez y al costo de los recursos naturales -el marfil por ejemplo- la innovación hoy es, en parte, una respuesta a la escasez potencial y al costo del petróleo: la inestabilidad persistente en las principales regiones productoras de petróleo mundiales termina en precios inestables del petróleo crudo. Más aún, la explotación del petróleo está vinculada a una variedad de males ambientales globales, desde el cambio en el clima hasta la contaminación de los océanos.

Las preocupaciones acerca de la salud humana son otra potente fuerza que impulsa el desarrollo de los plásticos. Según los centros para el control de enfermedades de los Estados Unidos, todos nosotros cargamos con un "agobio corporal" de toxinas, incluyendo componentes y aditivos plásticos que se incorporan al plástico para cumplir con criterios tales como flexibilidad y resistencia al fuego. En la última década, los científicos han demostrado que algunos de estos químicos son capaces de controlar el código genético para disparar una gama de efectos físicos y de comportamiento. Por ejemplo, en abril del 2003, genetistas de la Universidad Case Western Reserve mostraron que el bisfenol A, -que se usa en la manufactura de plásticos duros, transparentes, incluyendo recipientes para almacenar y cocinar alimentos en el microondas, biberones y sellantes de dientes que ayudan a prevenir las caries-interrumpe la forma en la que los cromosomas se alinean en los óvulos de 
ratones, generando la aneuploidía ${ }^{4}$, causa principal de abortos en los humanos y del síndrome de Down.

Las preocupaciones por la salud han llevado a nuevas regulaciones y acciones preventivas en muchas partes del mundo. En octubre de 2004, la Unión Europea prohibió permanentemente el uso de los ftalatos $^{5}$-aditivo que hace maleable el PVC (Policloruro de vinilo) - en juguetes de niños y en cosméticos. Los ftalatos se usan en aparatos para la dentición de los bebés, cortinas de baño y suministros médicos tales como bolsas para sangre y tubos, $y$ ayudan a que los cosméticos penetren en la piel. Los ftalatos han sido relacionados con daños en el hígado y riñones y malformaciones genéticas en infantes. Aunque en los Estados Unidos no los han prohibido, los fabricantes de juguetes, especialmente Mattel, en 1999 anunciaron que no fabricarían productos que los contuvieran.

Los deshechos son otro tema crucial. Hay países en la Unión Europea y Asia que están desarrollando regulaciones que obligan a las compañías a ser más responsables en el ciclo completo de sus productos. Además, las regulaciones de la UE introducirán requerimientos en forma paulatina para que las compañías reciban los productos de nuevo al final de su vida útil, lo cual procura que los deshechos y la disposición de toxinas, sea un asunto del fabricante, no del consumidor.

Para la industria norteamericana de tapetes, basada, en la mayoría de sus productos, en materiales plásticos notoriamente persistentes en los rellenos sanitarios, los deshechos se han vuelto un problema serio. En 1990, la industria firmó un acuerdo con la Agencia de Protección del Medio Ambiente (EPA) de los Estados Unidos para comenzar a incorporar más contenido reciclado en sus productos e investigar diseños más verdes.

\section{LA RESPUESTA DEL INNOVADOR}

La innovación puede ser, en gran medida, una respuesta a la regulación; el gobierno y la industria pueden ser socios en la sostenibilidad, como demuestran los ejemplos anteriores. Algunos innovadores, sin embargo, tienen la esperanza de ir más allá de una posición reactiva. Se están preguntando, ¿qué tal si, en lugar de vigilar toxinas y deshechos, produjéramos materiales que hicieran innecesarias esas regulaciones? Observemos al fabricante inglés de los biberones, el doctor Brown, que se inspiró en los estudios acerca del bisfenol $\mathrm{A}^{6}$ para diseñar un biberón que no incluyera esa sustancia. Consideremos también a Cargill Dow, una

4 Aneuploidía. Número anómalo de cromosomas que no constituyen un múltiplo exacto de un número haploide. Algunos ejemplos son la trisomía D, la trisomía E, el síndrome de Down y los síndromes XXX, XXY y XXYY. En inglés Aneuploidy.

5 Ftalatos. Este nombre comprende una amplia familia de compuestos. Entre los más comunes se encuentran: el dimetilftalato (DMF), el dietilftalato (DEF), el dibutilftalato, el bencilbutilftalato (BBF), el di (2etilhexil)ftalato (DEHF) (C24H38O4), y el dioctilftalato (DOF).

6 Estrógenos artificiales. El bisfenol A se encuadra en la categoría de los denominados estrógenos artificiales o, como se les ha venido denominando más recientemente, disruptores endocrinos. En general, se trata de productos químicos que mimetizan la acción de los estrógenos naturales (por ello se llaman también xenoestrógenos) y que interfieren en la acción del ciclo hormonal natural.

7 Joint Venture se define como la sociedad en participación, sociedad temporal, asociación de empresas, asociación temporal de empresas, unión temporal de empresas; El joint-venture es un anglicismo muy usado en contratos. Significa asociación de empresas; emprendimiento conjunto. 
sociedad temporal (joint venture) ${ }^{7}$ salida de las dos compañías matrices, que fabrica un plástico de maíz (ácido poliláctico ${ }^{8}$ o PLA) usado en productos que van desde recipientes para alimentos hasta autopartes. El PLA es completamente biodegradable y su principal insumo, el maíz, está ampliamente disponible. PLA tiene demanda en Japón, Taiwan, y la UE, regiones que reconocen la importancia de la independencia del petróleo y están atentas a los temas de deshechos y salud publica.

Shaw Industries, una compañía que está imponiendo nuevos estándares para la sostenibilidad en el negocio de pisos, es otro ejemplo de innovación de punta en plásticos. En el 2003 la compañía lanzó un tapete con el innovador sistema de respaldo "ecowor $x$ ", que usa poliolefinas ${ }^{10}$ como su ingrediente primario. El producto no solo es preferible desde el punto de vista de la salud y el medio ambiente, sino que requiere $40 \%$ menos contenido y es completamente reciclable. Para diciembre del 2004, Shaw se comprometió a dejar de emplear el PVC debido a sus muy conocidos negativas consecuencias sobre el medio ambiente y la salud. La compañía también planea instituir un sistema de reciclaje de tapetes para luego usar los materiales en nuevos productos. Siendo el fabricante de tapetes más grande del mundo, Shaw puede mover mercados. Y Shaw no está solo en la industria. La atención temprana frente al síndrome del edificio enfermo (causado por emisión gaseosa de toxinas provenientes de los materiales de construcción y del diseño interior) y el liderazgo de Ray Anderson en Interface Carpet, han puesto a esta industria a la cabeza del movimiento por la sostenibilidad.

Este movimiento, sin embargo, no está siendo liderado solamente por los propios fabricantes de materiales. Kaiser Permanente ${ }^{11}$, empresa prestadora de servicios de salud (HMO) más grande de los Estados Unidos, ha establecido una estrategia de compras que no sólo cumple con especificaciones de desempeño, sino que busca evitar los deshechos y efectos adversos en salud. Kaiser pregunta a sus proveedores acerca del contenido de los productos que fabrica: tapetes, bolsas para sangre, termómetros y tubos. El punto de vista de la firma es que si los profesionales de la salud están usando bolsas para sangre que contienen dosis de químicos que interrumpen la producción de substancias endocrinas a bebés prematuros,

8 El ácido poliláctico (PLA), es un biopolímero termoplástico cuya molécula precursora es el ácido láctico. Debido a su biodegradabilidad, propiedades de barrera y biocompatibilidad, este biopolímero ha encontrado numerosas aplicaciones.

9 Ecoworx TM: Base 100\% libre de PVC, compuestos termoplásticos de poliolefina y una capa de refuerzo central de fibra de vidrio. Ofrece mayor durabilidad y estabilidad para el alto tránsito. La baldosa da mayor flexibilidad en el manejo del material, colocación en espacios ocupados y menor desperdicio. Es una marca.

10 Poliolefinas. Denomincación general para los polímeros, los cuales se obtienen por polimerización radicalizada de olefinas. Los ejemplos más importantes son: PE, PP, PB, PIB, etc. Las poliolefinas tienen en común la falta de grupos funcionales y por lo tanto, poca polaridad. Por eso los revestimientos sobre superficies de poliolefinas sin preparación previa tienen una mala adhesión. La adhesion de la mayoría de los recubrimientos sobre poliolefinas se puede mejorar considerablemente mediante tratamientos con plasma. casi siempre con oxígeno como gas plasmágeno.

11 Kaiser Permanente es una de las más grandes y exitosas OCS (organizaciones para el cuidado de la salud) de los Estados Unidos. 
no están cumpliendo con su compromiso de "no hacer daño". Kaiser está comprometido en usar su poder de compra para apoyar su misión de promover la salud pública y el bienestar. La compañía trabaja con los proveedores existentes para desarrollar nuevos productos que cumplan con especificaciones amplias de desempeño y ciclo de vida. Y sus proveedores están dispuestos a tomar el riesgo de desplegar una nueva línea de productos porque saben que Kaiser será un cliente valioso.

El tipo de fuerzas que están detrás del crecimiento de los plásticos sostenibles son las que siempre han inspirado a los empresarios e innovadores. Las crecientes preocupaciones en torno a los plásticos derivados de combustibles fósiles representan una oportunidad para rediseñar plásticos a lo largo de la cadena de suministro e impulsan una nueva generación de productos al mercado. Las innovaciones que vemos ahora en la industria de los plásticos representan la historia clásica del empresario, en la que las compañías líderes e individuos ven oportunidades donde otros sólo ven problemas.
BATTEN BRIEFING.

Translated and published with permission by the Batten Institute at the Darden Graduate School of Business Administration, University of Virgina.

\section{BATTEN BRIEFINGS.}

Traducido y publicado con el permiso del Instituto Batten en el Darden Graduate School of Business Administration, University de Virgina. 